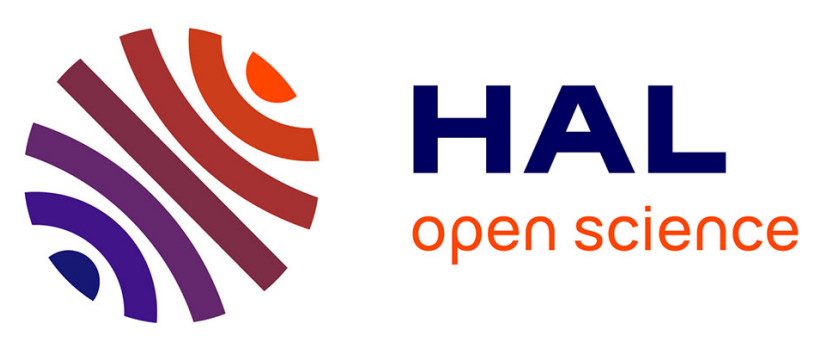

\title{
Theoretical Study of the Nonlinear Anelastic Internal Friction Peaks Associated with Diffusion of Solute Atoms in Dislocation Core
}

Q. Fang, T. Kê

\section{- To cite this version:}

Q. Fang, T. Kê. Theoretical Study of the Nonlinear Anelastic Internal Friction Peaks Associated with Diffusion of Solute Atoms in Dislocation Core. Journal de Physique IV Proceedings, 1996, 06 (C8), pp.C8-273-C8-276. 10.1051/jp4:1996858 . jpa-00254666

\section{HAL Id: jpa-00254666 https://hal.science/jpa-00254666}

Submitted on 1 Jan 1996

HAL is a multi-disciplinary open access archive for the deposit and dissemination of scientific research documents, whether they are published or not. The documents may come from teaching and research institutions in France or abroad, or from public or private research centers.
L'archive ouverte pluridisciplinaire HAL, est destinée au dépôt et à la diffusion de documents scientifiques de niveau recherche, publiés ou non, émanant des établissements d'enseignement et de recherche français ou étrangers, des laboratoires publics ou privés. 


\title{
Theoretical Study of the Nonlinear Anelastic Internal Friction Peaks Associated with Diffusion of Solute Atoms in Dislocation Core
}

\author{
Q.F. Fang and T.S. Kê
}

\begin{abstract}
Laboratory of Internal Friction and Defect in Solids, Institute of Solid State Physics, Chinese Academy of Sciences, 230031 Hefei, China
\end{abstract}

\begin{abstract}
Based on the dislocation kink atmosphere model, the longitudinal and transverse core diffusion equation of solute atoms was formulated. The relaxation time and the relaxation strength associated with the diffusion processes under quasi-static stress were computed by solving the diffusion equation analytically, and were found to be nonlinear with respect to the applied stress. The theoretical internal friction curves versus temperature and strain amplitude were calculated by solving the diffusion equations with numerical method and manifestation of these curves conform to the experimental results previously obtained.
\end{abstract}

\section{INTRODUCTION}

In a previous paper by $\mathrm{Ke}[1]$, the mathematical analysis of the dislocation core diffusion of point defect by Winkler-Gniewek et al. (designated as the WG theory)[2] on the basis of a double-loop string model were described. It was shown that the theoretical internal friction curves derived by WG conform, in a certain extent, to the manifestations of the anomalous internal friction peaks appearing in cold-worked Al-Cu and $\mathrm{Al}-\mathrm{Mg}$ specimens around room temperature which were attributed to the dislocation core diffusion. However, it was pointed out that the physical picture on the basis of the string model is not clear especially for the transverse core diffusion. In this paper the problem of dislocation core diffusion will be treated on the basis of the kink model, and the theoretical curves of internal friction and modulus defect will be compared with experimental results.

\section{THEORETICAL CONSIDERATION}

\subsection{Distribution function of the solute atom on an ensemble of kink chain}

A series of kinked dislocations (kink chains) should exist in a cold-worked specimen. We may consider only the geometrical kink chains. Every kink in each kink chain can interact with solute atoms, so that one solute atom can be situated on each kink. However, to simplify the theoretical manipulation, we may consider the case when each kink chain contains only one solute atom. It can be shown that, the conclusion is similar in the case when many solute atoms are involved. Without the loss of generality, we can assume that the solute atom is situated on the right hand of the middle point of the kink chain with the coordinate $\mathrm{x}$ along the direction of the Peierls valley. The kink chain is thus divided into two segments with lengths $\mathrm{L}+\mathrm{x}$ and $\mathrm{L}-\mathrm{x}$, where $2 \mathrm{~L}$ is the length of the kink chain projected on the $\mathrm{x}$-axis. Thus the position of the solute atom is expressed by $(x, y)$ with the $y$-axis perpendicular to the direction of the Peierls valley and the origin of $y$ coordinate is on the dotted skew line as shown in Fig.1. Assume that $\rho(x, y, t)$ dxdy represents the fraction of the number of kink chains on which the solute atom is situated between $x \rightarrow x+d x$ and $y \rightarrow y+d y$ compared with the total number of an ensemble of kink chains with a length $2 \mathrm{~L}$ contained in the specimen, 
then by definition we have

$$
\int_{-L}^{+L} d x \int_{-\infty}^{+\infty} d y \rho(x, y, t)=1
$$

Consequently, $\rho(x, y, t)$ represents the probability density or the distribution function that the solute atom appears at the position $(x, y)$ among an ensemble of similar kink chains.

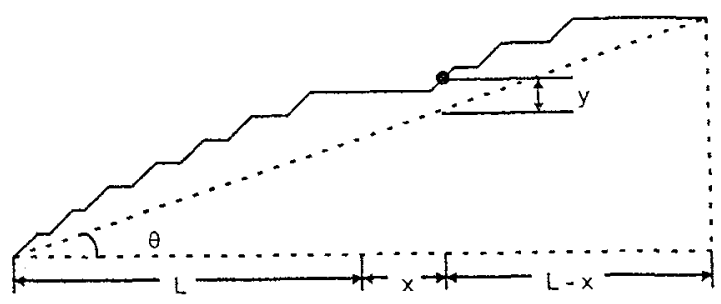

Fig.1: Schematic diagram of a geometrical kink chain with a solute atom situated at the middle of one kink under the action of external stress

\subsection{Diffusion equation of the solute atom along dislocation core}

Considering the interaction with the nearest neighboring kinks and the potential energy established by the applied stress field, the total energy increment of the kink chain in stressed state compared with the unstressed state is shown to be[3]:

$$
W=-\frac{(\sigma b h)^{2} L}{P} x^{2}+\frac{P L y^{2}}{h^{2}\left(L^{2}-x^{2}\right)}-\sigma b L y+\text { constant }
$$

in which $P=\frac{G b^{2} h^{2}}{4 \pi} \gamma, \gamma \approx 1, b$ is the Burgers vector, $h$ is the height of the kink, $\sigma$ is the external stress, and $\mathrm{G}$ is the shear modulus.

It can be seen from Fig. 1 that the coordinate y of the solute atom changes during the sidewise motion of the kink it attached, while $y+(L+x) \tan \theta$ remains on the same level. The longitudinal and transverse force along $\mathrm{x}$ and $\mathrm{y}$ direction exerted on the solute atom are, thus, respectively

$$
\begin{gathered}
F_{L}=-\frac{\partial W}{\partial x}-\frac{\partial W}{\partial y} \frac{\partial y}{\partial x}=\frac{2(\sigma b h)^{2} L x}{P}-\frac{\beta x y^{2}}{L^{2}-x^{2}}+\beta\left(y-y_{0}\right) \tan \theta \\
F_{T}=-\frac{\partial W}{\partial y}=-\beta\left(y-y_{0}\right), \\
\text { where } \quad y_{0}=\frac{\sigma b h^{2}\left(L^{2}-x^{2}\right)}{2 P}, \quad \beta=\frac{2 P L}{h^{2}\left(L^{2}-x^{2}\right)}
\end{gathered}
$$

Under the action of the forces $\mathrm{F}_{\mathrm{L}}$ and $\mathrm{F}_{\mathrm{T}}$, the solute atom will diffuse and the change of its distribution function will be governed by the following equation:

$$
\frac{\partial \rho}{\partial t}=D_{T} \frac{\partial}{\partial y}\left(\frac{\partial \rho}{\partial y}-\frac{F_{T}}{k T} \rho\right)+D_{L} \frac{\partial}{\partial x}\left(\frac{\partial \rho}{\partial x}-\frac{F_{L}}{k T} \rho\right)
$$

where $D_{L}$ and $D_{T}$ are the longitudinal and transverse diffusion coefficients respectively.

When the solute atom is situated on the extremities of the kink, then during the sidewise motion of the kink, the solute atom can diffuse along the straight dislocation segment (pure longitudinal diffusion) or diffuse upward through a series of the sidewise motion of the kink (pure transverse diffusion). The former case gives rise to the $P_{0}$ peak and the latter case gives rise to the $P_{1}{ }^{\prime \prime}$ peak[3]. The internal friction peak $P_{1}{ }^{\prime}$ however, is attributed to the longitudinal core diffusion of the solute atom situated on the middle of the kink as shown in Fig. 1. In this paper, we will discuss only the $P_{1}^{\prime}$ peak.

In the case of $P_{1}$ ' peak, the diffusion equation and its initial and boundary condition can be written in the form[3]:

Diffusion equation: $\quad \frac{\partial \rho_{L}}{\partial t}=\frac{D_{L}}{L^{2}} \frac{\partial}{\partial \xi}\left(\frac{\partial \rho_{L}}{\partial \xi}-a^{2} \xi \rho_{L}\right)$ 
Boundary condition: $\left[\frac{\partial \rho_{L}}{\partial \xi}-\alpha^{2} \rho_{\bar{L}}\right]_{\xi=+1} \doteq\left[\frac{\partial \rho_{L}}{\partial \xi}+\alpha^{2} \rho_{L}\right]_{\xi=-1}=0$

Normalized condition: $\quad \int_{-1}^{+1} L \rho_{L}(\xi, t) d \xi=1$

Initial condition: $\quad \rho_{L}(\xi, 0)=\frac{1}{2 L}$

where $\quad \alpha^{2}=\frac{3(o b h)^{2} L^{3}}{4 P k T}, \quad \xi=\frac{x}{L}$,

and $\mathrm{k}$ is Boltzmann constant, $\mathrm{T}$ is the absolute temperature, $\mathrm{t}$ is the time, $\rho_{\mathrm{L}}(\xi, \mathrm{t})$ is the longitudinal distribution function, and relates to the total distribution function $\rho(\xi, y, t)$ :

$$
\rho(\xi, y, t)=\rho_{L}(\xi, t) \sqrt{\frac{\beta}{2 \pi k T}} \exp \left[-\frac{\beta\left(y-y_{0}\right)^{2}}{2 k T}\right]
$$

The anelastic strain can be calculated by

$$
\varepsilon_{A}(t)=\frac{3 \Lambda \sigma(b h)^{2} L^{2}}{4 P}\left[\frac{7}{9}+\int_{-1}^{+1} \rho_{L}(\xi, t) \xi^{2} d \xi\right]
$$

\subsection{Relaxation time, relaxation strength, internal friction and modulus defect of the $P_{1}$ ' peak}

If the external stress is quasi-static, i.e. $\sigma=0$ when $t \leq 0$ and $\sigma$ is constant when $t>0$, the analytical solution of the equations (6-9) can be obtained as a function of $x$ and $t$ with the relaxation time equal to

$$
\tau=\frac{L^{2}}{D_{L}} \frac{1}{\pi^{2}+2 \alpha^{2}}
$$

where $\alpha^{2}=\frac{3(\sigma b h)^{2} L^{3}}{4 P k T}$

The relaxation strength can be calculated from the anelastic strain produced when the distribution function changes from the initial state (at $t=0$ ) to the final stationary state (at $t=\infty$ ):

$$
\Delta=\frac{\pi \Lambda L^{2}}{3+8 \pi \Lambda L^{2}}\left[9 C L \int_{-1}^{+1} \xi^{2} \exp \left(\alpha^{2} \xi^{2}\right) d \xi-1\right],
$$

where $\wedge$ is the effective dislocation density and $\mathrm{C}$ is the normalized parameter.

To calculate the internal friction and modulus defect under a periodical external stress, $\sigma=\sigma_{0} \sin \omega t$, the partially differential equation (6) with its initial and boundary conditions (7-9) must be solved numerically, and then the total strain as a function of time (12) can be obtained. The internal friction and modulus defect can thus be computed by the following equations:

Internal friction: $\quad Q^{-1}=\frac{\Delta W}{2 \pi W}=\frac{-G}{\pi \sigma_{0}^{2}} \oint \varepsilon d \sigma=\frac{-G}{\pi \sigma_{0}} \sum_{j=0}^{2 \pi / \Delta t} \cos (j \Delta t) \varepsilon(j \Delta t) \Delta t$.

The stored energy: $\quad W=\int_{0}^{\pi / 2} \sigma d \varepsilon=\sigma_{0} \varepsilon\left(\frac{\pi}{2 \Delta t}\right)-\sigma_{0} \sum_{j=0}^{\pi / 2 \Delta t} \cos (j \Delta t) \varepsilon(j \Delta t) \Delta t$.

Modulus defect: $\quad \frac{\Delta M}{M}=\frac{2 G W}{\sigma_{0}^{2}}-1$.

where $\Delta t$ is the integration step of the time variable $\mathrm{t}^{\prime}=\omega \mathrm{t}, \quad \varepsilon(t)=\varepsilon_{A}(t)+\frac{\sigma}{G}$ is the total strain.

\section{RESULTS AND DISCUSSION}

By numerical calculation, we take $\Lambda \mathrm{L}^{2}=0.01, \mathrm{f}=1 \mathrm{~Hz}, \mathrm{~Gb}^{3}=5 \mathrm{eV}, \mathrm{L}=10^{3} \mathrm{~b}$. The longitudinal diffusion coefficient $\mathrm{D}_{\mathrm{L}}$ has the form $D_{L}=D_{L 0} \exp \left(-\frac{H}{k T}\right)$ with $\mathrm{H}=0.5 \mathrm{eV}[4]$, and $\mathrm{D}_{\mathrm{L}, 0}$ is so selected that the 
temperature internal friction peak locates at $340 \mathrm{~K}$ when $\sigma=0$ and $\mathrm{f}=1 \mathrm{~Hz}$.

Fig. 2 shows the variation of the relaxation time $\tau / \tau_{0}\left(\tau_{0}\right.$ is the relaxation time at $\left.\sigma=0\right)$ and the relaxation strength $\Delta$ as a function of strain amplitude $\varepsilon_{0}$ at $\mathrm{T}=340 \mathrm{~K}$. It can be seen that $\tau / \tau_{0}$ decreases and $\Delta$ increases with the increase of $\varepsilon_{0}$. This result is consistent with the experimental result of $\mathrm{P}_{1}$ peak in $\mathrm{Al}-\mathrm{Cu}$ alloy[5].

In Fig. 3 are given the variations of internal friction and modulus defect versus temperature (a) and strain amplitude (b). As the strain amplitude increases, the temperature internal friction peak shifts toward higher temperature as shown in Fig. 3a. The modulus defect increases with the

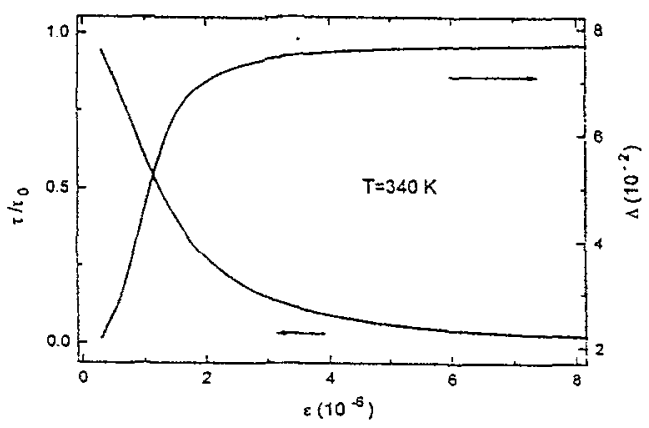

Figure 2: Variation of the normalized relaxation time $\tau / \tau_{0}$ and the relaxation strength $\Delta$ versus strain amplitude increase of temperature (a) or strain amplitude.(b).

The strain amplitude internal friction peak shown in Fig. 3b shifts toward lower strain amplitude as the temperature increases, in consistence with the experimental results of $\mathrm{P}_{1}$ ' peak in $\mathrm{Al}-\mathrm{Mg}$ solid solution[6].

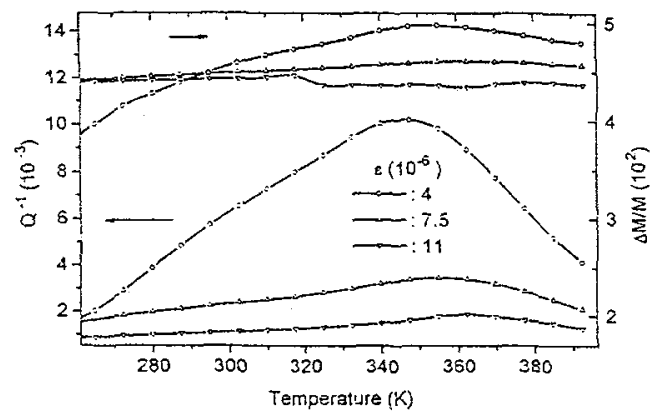

a)

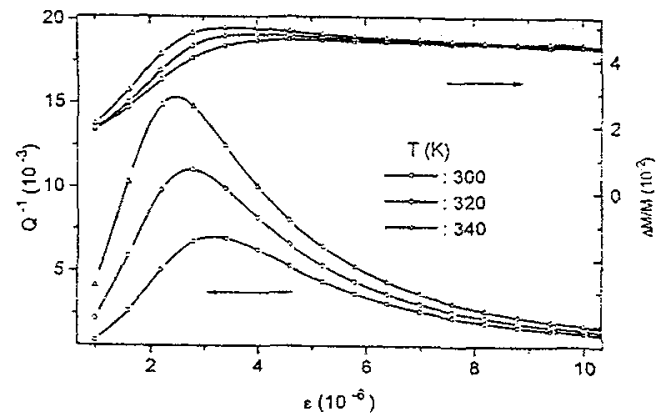

b)

Figure 3: Internal friction and modulus defect curves of the $P_{1}$ ' peak versus temperature (a) and strain amplitude (b). In (a), curves 1-3 correspond to the strain amplitude $4,7.5$ and $11 \times 10^{-6}$ respectively; in (b), curves $1-3$ correspond to the temperature 300,320 and $340 \mathrm{~K}$ respectively.

\section{Acknowledgments}

This work has been subsidized by the National Natural Science Foundation of China. One of the authors (Q.F. Fang) gratefully acknowledges the financial support of K.C. Wong Education Foundation, Hong Kong.

\section{References}

[1] Ke T.S., phys. sat. sol. (a) 155(1996): 83

[2] Winkler-Gniewek W., Schlipf J, Schindlmayer S. in: Proc. ICIFUACS-5 (ed. Lenz D, Lücke K), Springer-Verlag, Berlin, 1975, Vol. II , 246

[3] Fang Q.F. and Ke T.S., communicated to phys. sat. sol. (a) (1996)

[4] Tan Q and Kê T.S., phys. stat. sol. (a) 122(1990): K25

[5] Zhu A.W., Yuan L.X and Kê T.S., phys. stat. sol. (a) 135(1993): 151

[6] Kê T.S., Tan Q. and Fang Q.F., phys. stat. sol. (a) 103(1987): 421 